\title{
Évaluation internationale et régulation de la politique éducative : le cas du Maroc dans l'enquête TIMSS 2015
}

\section{Abdelhak Ghouli}

\section{(2) OpenEdition}

\section{Journals}

Édition électronique

URL : https://journals.openedition.org/ries/8872

DOI : $10.4000 /$ ries. 8872

ISSN : 2261-4265

Éditeur

France Education international

\section{Édition imprimée}

Date de publication : 1 décembre 2019

Pagination : 16-19

ISBN : 9782854206258

ISSN : $1254-4590$

\section{Référence électronique}

Abdelhak Ghouli, «Évaluation internationale et régulation de la politique éducative : le cas du Maroc dans l'enquête TIMSS 2015 ", Revue internationale d'éducation de Sèvres [En ligne], 82 | décembre 2019, mis en ligne le 01 décembre 2021, consulté le 15 janvier 2022. URL : http://journals.openedition.org/ ries/8872 ; DOI : https://doi.org/10.4000/ries.8872

Ce document a été généré automatiquement le 15 janvier 2022.

(c) Tous droits réservés 


\title{
Évaluation internationale et régulation de la politique éducative : le cas du Maroc dans l'enquête TIMSS 2015
}

\author{
Abdelhak Ghouli
}

1 Les enquêtes internationales sur les acquis des élèves, notamment TIMSS ${ }^{1}$, montrent que des disparités scolaires résident dans les différents systèmes éducatifs étudiés et que cette variance s'explique d'une part par des facteurs sociétaux, dont l'impact parait limité, et d'autre part par les politiques éducatives conduites (Gorard et Smith, 2004 ; Mons, 2007). L'exploitation des bases de données des enquêtes internationales sur les acquis des élèves donne lieu à des analyses secondaires mettant en relation les performances des élèves avec les structures éducatives ainsi que les moyens empiriques de questionner les choix politiques. Les résultats de TIMSS 2015 permettent d'évaluer l'efficacité et l'équité des systèmes éducatifs, tout en essayant de déterminer les facteurs scolaires et extrascolaires susceptibles d'influencer les apprentissages.

2 Avec une moyenne de 376 points en mathématiques et de 390 en sciences, les scores des élèves marocains montrent un sérieux problème d'acquisition des connaissances et de maîtrise des compétences. Plusieurs hypothèses peuvent être formulées pour expliquer ces résultats: i) surcharge des programmes dominés par l'aspect théorique; ii) méthode d'enseignement focalisée sur l'accumulation des connaissances plutôt que sur le développement des aptitudes des élèves; iii) modernisation insuffisante des méthodes et des outils pédagogiques; iv) persistance d'une uniformité de l'enseignement devenue incompatible avec la diversité des situations et la nécessité d'adaptation à des réalités différentes.

3 Au Maroc, ce constat d'un faible niveau de maitrise de compétences de base a mené, depuis 2017, à améliorer les pratiques de l'enseignement en classe en révisant les plans de leçons avec un examen attentif aux difficultés réelles des élèves ainsi qu'au développement de matériels d'enseignement et d'apprentissage supplémentaires. En 
outre, un investissement important a été déployé en matière de rénovation pédagogique, de révision des curricula, de restructuration du cycle et de diversification et de production de nouveaux manuels scolaires afin de les mettre en adéquation avec les programmes d'études et les normes internationales en matière de socles de connaissances de base.

4 La valeur ajoutée de l'analyse des résultats de TIMSS a incité les décideurs marocains à remédier à cette situation ; l'autorité centrale et le niveau déconcentré ont mis en place un dispositif de pilotage pédagogique. Pour les établissements scolaires, il s'agit d'exploiter la nouvelle composante du système d'information de suivi des élèves (MASSAR), afin d'identifier les élèves en difficulté et les items non réussis pour programmer le soutien scolaire.

Figure 1 : Distribution normale des scores des élèves en mathématiques au Maroc

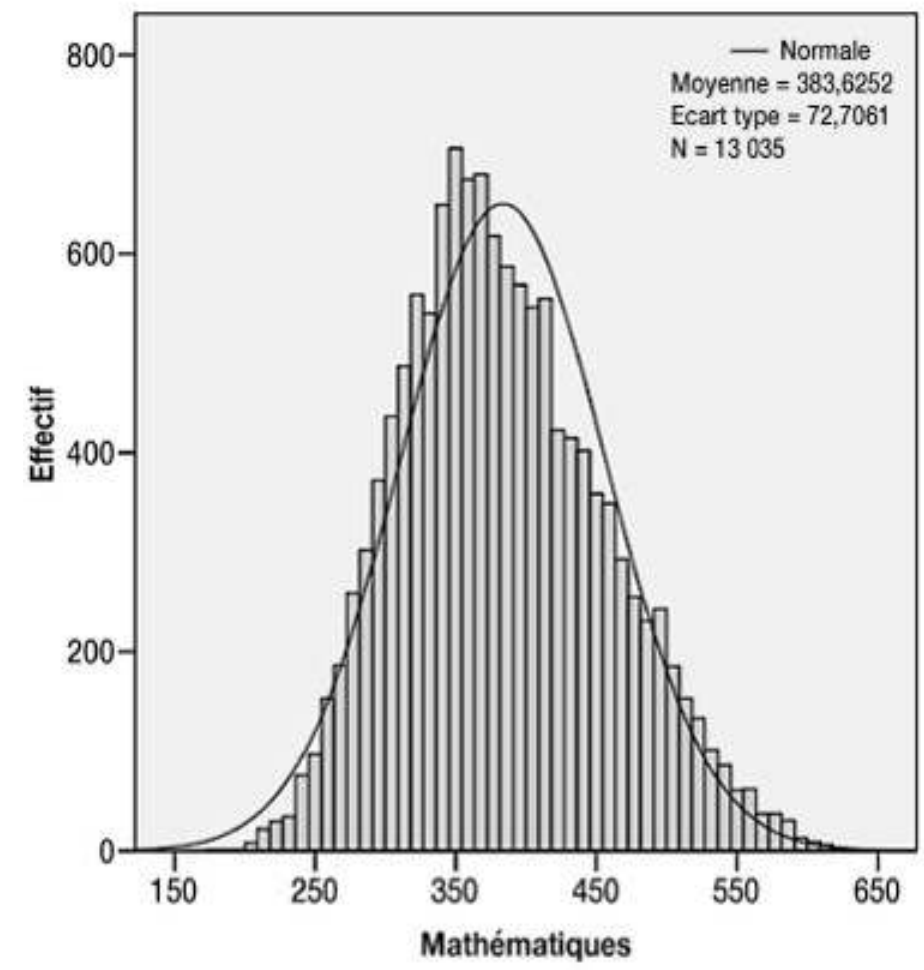

Source : base de données TIMSS 2015

\section{Amélioration de la politique de gestion locale}

5 Au Maroc, les chefs d'établissement ne disposent pas de l'autonomie administrative ou budgétaire leur permettant de prendre des mesures pour résoudre les problèmes spécifiques d'un établissement scolaire et la communauté scolaire ne se sent pas responsable des résultats des performances, étant donné que sa participation, son appropriation des solutions et sa capacité à prendre des initiatives demeurent extrêmement limitées.

6 En dehors de quelques établissements exceptionnels où les élèves obtiennent des scores moyens compris entre 600 et 800 points, dans la plupart des établissements, les scores obtenus en mathématiques se situent dans une moyenne comprise entre 200 et 
400 points. Ce type d'analyse de résultats a encouragé les responsables du système éducatif marocain à introduire et améliorer de plus en plus des politiques de gestion locale appuyées sur ce qu'on appelle le projet d'établissement. Les écoles établissent leur diagnostic, se fixent des objectifs à atteindre et cherchent les moyens pour les réaliser. On s'installe alors nécessairement dans une logique de transparence et de reddition des comptes.

7 D'ailleurs, il s'agit bien souvent d'un maillon faible des systèmes éducatifs. Il importe que des dispositions soient identifiées et concrètement mises en place pour que les ressources mobilisées et les politiques éducatives nationales aboutissent effectivement jusqu'au niveau des établissements scolaires et que ces derniers transforment efficacement en résultats d'apprentissages les ressources dont ils disposent.

\section{L'évaluation internationale TIMSS et les choix politiques}

8 L'âge légal des élèves de $8^{\mathrm{e}}$ année au Maroc étant de 13 ans, le retard scolaire enregistré peut être justifié par plusieurs hypothèses, à savoir l'insertion des enfants à l'école de la deuxième chance, l'entrée tardive des enfants dans les écoles et également le phénomène du redoublement. En tenant compte des statistiques officielles sur le redoublement au Maroc, nous favorisons en premier lieu l'hypothèse du redoublement comme facteur principal dans cette analyse.

9 En examinant les résultats de TIMSS 2015, on constate l'absence de redoublement dans le système éducatif des pays classés parmi les dix premiers dans TIMSS 2015. Les redoublants sont souvent démotivés. Il ne s'agit pas ici de remettre en cause la pratique $\mathrm{du}$ redoublement dans son ensemble. Cependant, des mesures d'accompagnement sont nécessaires pour une plus grande efficacité.

10 Avec un score moyen de 360 points contre 406 pour les élèves de moins de 14 ans, les élèves âgés de plus de 14 ans, ayant donc redoublé, ont un score nettement inférieur à ceux qui n'ont pas redoublé. On peut donc s'interroger sur le redoublement et sur son efficacité. Le redoublement n'apporte pas les bénéfices escomptés sur le plan des acquisitions. Cela confirme d'une part l'inefficacité du recours au redoublement comme mesure de remédiation aux difficultés scolaires, destinée à gérer les difficultés des élèves et leurs différences de rythme d'apprentissage dans l'acquisition des compétences de base, tout en augmentant, d'autre part, le risque d'échec et de déperdition.

11 Sur un autre registre, les résultats du Maroc à TIMSS 2015 montrent que les élèves qui utilisent l'ordinateur dans les établissements scolaires ont des résultats faibles par rapport aux autres. Cela doit nous interpeller sur l'exploitation des salles multimédias des établissements scolaires et sur la place que nous réservons aux TIC dans l'enseignement.

12 Quand ceux qui utilisent l'ordinateur chaque jour obtiennent un score de 360 contre 405 pour ceux qui ne l'utilisent jamais, et que les résultats diminuent en fonction de l'intensité de son usage, il apparait certain qu'il ne suffit pas d'équiper les établissements scolaires par des ordinateurs mais qu'il faudra mettre en place des curricula assurant l'accès aux TIC au plus grand nombre d'élèves, afin que ces derniers intègrent leur utilisation dans le développement de leurs compétences dans les 
diverses disciplines. La Vision stratégique ${ }^{2}$ dans son article 105, préconise de renforcer l'intégration de ces technologies à l'école en vue de la promotion de la qualité des apprentissages, notamment en ce qui concerne la conception et la préparation des curricula, des programmes, des matières et au cours de leur mise en œuvre. Ainsi l'utilisation des logiciels et des ressources numériques interactives dans l'ensemble du processus pédagogique devrait cibler l'auto-apprentissage, la recherche et la diversification des sources d'apprentissage.

L'évaluation de TIMSS n'est pas une finalité en soi ; elle est à considérer comme un outil d'évaluation et de repérage des réussites et des difficultés des programmes nationaux. Ce type d'évaluation consiste à mettre à la disposition des responsables politiques des systèmes d'éducation et de formation les grandes orientations nécessaires à la prise de décision visant le développement du processus d'enseignement et d'apprentissage, des curricula et des programmes ainsi que l'amélioration des conditions de l'enseignement et le perfectionnement des outils didactiques et pédagogiques. Les résultats de TIMSS permettent également de mettre en évidence les mesures urgentes et prioritaires à mettre en œuvre comme réponse au faible niveau des apprentissages constatés dans les items de l'évaluation.

Une éducation de qualité reste un objectif fort dans la politique éducative marocaine. L'évaluation TIMSS 2015 montre, d'une part, que les élèves ont encore de sérieuses difficultés en sciences et en mathématiques et, d'autre part, que les conditions pour une éducation de qualité ne sont pas encore complètement réunies. La Vision stratégique 2015- 2030 est confrontée à plusieurs défis, à savoir l'amélioration du taux d'achèvement, la réduction des disparités selon le genre et la zone géographique ainsi que l'amélioration de la formation initiale et continue des enseignants.

\section{BIBLIOGRAPHIE}

BROADFOOT P. (2000). « Un nouveau mode de régulation dans un système décentralisé : l'État évaluateur ». Revue française de pédagogie, $\mathrm{n}^{\circ}$ 130, p. 43-55.

GORARD S. et SMITH E. (2004). «An international comparison of equity in education systems ». Comparative Education, $\mathrm{n}^{\circ}$ 40, p. 15-28.

MONS N. (2007). «L'évaluation des politiques éducatives : apports, limites et nécessaire renouvellement des enquêtes internationales sur les acquis des élèves ». Revue internationale de politique comparée, vol. 14, 3, p. 385-397.

\section{NOTES}

1. L'enquête internationale TIMMS (Trends in International Mathematics and Science Study), menée par l'IEA (International Association for the Evaluation of Educational Achievement), évalue les acquis scolaires des mathématiques et des sciences. ( $N d l R$ ) 
2. Voir : Rahma Bourqia (2016). «Repenser et refonder l'école au Maroc: la Vision stratégique 2015-2030 ", Revue internationale d'éducation de Sèvres, n 71, p. 18-24. [En ligne : DOI : 10.4000/ries. 4551]. (NdlR)

\section{INDEX}

\section{Index géographique : Maroc}

Palabras claves : matemáticas, repetición, evaluación del estudiante, encuesta internacional, TIMSS: Trends in International Mathematics and Science Study

Keywords : mathematics, grade repetition, student evaluation, international tests, TIMSS: Trends in International Mathematics and Science Study

Mots-clés : mathématiques, redoublement, évaluation des élèves, enquête internationale, TIMSS : Trends in International Mathematics and Science Study

\section{AUTEUR}

\section{ABDELHAK GHOULI}

Abdelhak Ghouli est chef de division de la planification au département de l'éducation nationale du Maroc et chercheur en sciences de l'éducation. Ses domaines de spécialité incluent, outre la planification, l'économie de l'éducation et notamment l'élaboration des comptes nationaux de l'éducation, de cadres de dépense à moyen terme (CDMT) ainsi que l'analyse de données coût/ efficacité et rendement d'investissement dans l'éducation. Courriel :

Abdelhak.GHOULI@men.gov.ma 